TITLE:

\title{
Duality for actions and coactions of measured groupoids on von Neumann algebras
}

$\operatorname{AUTHOR}(S)$ :

Yamanouchi, Takehiko

\section{CITATION:}

Yamanouchi, Takehiko. Duality for actions and coactions of measured groupoids on von Neumann algebras. 数理解析研究所講究録 1991, 751: 113-126

\section{ISSUE DATE:}

1991-05

URL:

http://hdl.handle.net/2433/82049

RIGHT: 


\title{
Duality for actions and coactions of measured groupoids on von Neumann algebras
}

\author{
Takehiko Yamanouchi \\ (山,内教层) \\ Department of Mathematics \\ University of California, Los Angeles \\ Los Angeles, CA 90024
}

\section{$\S 1$. Definitions}

In this note, we would like to discuss the theory of actions and coactions of measured groupoids on von Neumann algebras, which has been developed recently in author's dissertation at UCLA. Before we explain what we mean by actions and coactions of measured groupoids, we think that it is better to mention the history of groupoid actions on von Neumann algebras briefly. A notion of an action of a measured groupoid on a von Neumann algebra first appeared in the work of Jones and Takesaki [J\&T]. It appeared throught the classification of compact abelian group actions on semifinite injective factors. Their situation is the following: Starting from an action $\beta$ of a compact abelian group $G$ on a semifinite injective factor $\mathcal{R}$, they needed to analyze the dual structure $\mathcal{M}=\mathcal{R} \times{ }_{\beta} G$. For this purpose, they considered the central decomposition of $\mathcal{M}$ and then got an action of some measured groupoid associated with the dual action $\hat{\beta}$. In this case, von Neumann algebras appearing in the fibers in the central decomposition are all isomorphic. Hence they defined an action of a measured groupoid $\mathcal{G}$ by a Borel homomorphism $\alpha$ of $\mathcal{G}$ into 


\section{4}

the group of all automorphisms of one von Neumann algebra. Then, using this definition, Masuda defined a crossed product of an action of a measured groupoid on a von Neumann algebra. However, he did not discuss coactions of measured groupoids at all and, therefore, he could not show any duality. Now we observe that, in general, when we consider the central decomposition of a von Neumann algebra, we do not necessarily get isomorphic algebras in the fibers. This observation suggests that, in the definition of an action of a measured groupoid, we should allow ourselves to have non-isomorphic algebras if we would like to work in the right framework. We should also keep in mind that a groupoid is a small category with inverses. With these observation in mind, we state the definition of a groupoid action. In what follows, a measured groupoid is a second countable locally compact topological groupoid $\mathcal{G}$ that admits a faithful proper transverse function $\left\{\lambda^{x}\right\}_{x \in X}$ ( $X$ is the unit space of $\mathcal{G}$ ) and a semifinite transverse measure $\Lambda$ with a module $\delta$. Let $\Lambda_{\lambda}$ (or, simply $\mu$ ) denote the quasi-invariant measure on $X$ corresponding to $\left\{\lambda^{x}\right\}$ and $\Lambda$. Let $\nu$ be the measure on $\mathcal{G}$ obtained by integrating $\left\{\lambda^{x}\right\}$ with respect to $\mu$. We borrow notations related to groupoids, such as $s$ (=the source map), $r$ (=the range map), $\mathcal{G}^{x}$ and so on, from $[\mathbf{R a}]$.

Definition 1 (Actions). An action of $\mathcal{G}$ is a functor $\mathcal{F}$ from $\mathcal{G}$ into the subcategory of von Neumann algebras whose arrows are *-isomorphisms, such that, with $\mathcal{M}(x)=$ $\mathcal{F}(x)(x \in X)$ and $\alpha_{\gamma}=\mathcal{F}(\gamma)(\gamma \in \mathcal{G})$, we have the following conditions on them:

(i) the family $\left\{\mathcal{M}(x), L^{2}(\mathcal{M}(x))\right\}_{x \in X}$ forms a measurable field of von Neumann algebras over $(X, \mu)$, where $L^{2}(\mathcal{M}(x))$ is the canonical $L^{2}$-space of $\mathcal{M}(x)$ in the sense of Kosaki [K]. 


\section{5}

(ii) an algebraic groupoid representation $\gamma \in \mathcal{G} \mapsto u(\gamma)$ over $\left\{L^{2}(\mathcal{M}(x))\right\}_{x \in X}$ is measurable, where $u(\gamma)$ is the unique unitary from $L^{2}(\mathcal{M}(s(\gamma)))$ onto $L^{2}(\mathcal{M}(r(\gamma)))$ which implements the *-isomorphism $\alpha_{\gamma}$. (See [R] for the definition of a groupoid representation).

Before we proceed to the definition of a coaction of a measured groupoid, we would like to say a few words on the above mentioned Masuda's work. The reason why Masuda failed to discuss coactions of measured groupoids can be summerized in the follwing way. In the case of group actions, the existence of the dual coaction (see [N\&T] for its definition) on a crossed product by an action is essentially due to the Hopf-algebraic structure of a group algebra. However, to author's knowledge, no substitute for group algebras that admits a "Hopf-algebra-like" structure suitable for the groupoid setting has been dicovered in the case of groupoid actions. For the substitute, we look at the von Neumann algebra $\mathcal{R}(\mathcal{G})$ obtained from every measured groupoid $\left(\mathcal{G},\left\{\lambda^{x}\right\}, \Lambda, \delta\right)$ by P. Hahn's method, which we call the groupoid von Neumann algebra. To show that every groupoid von Neumann algebra possesses a Hopf-algebra-like structure, we need to recall some fundamental facts on the relative tensor product of Hilbert spaces over von Neumann algebras due to Sauvageot $[\mathbf{S 1}],[\mathbf{S 2}]$.

Let $\mathcal{H}_{1}$ and $\mathcal{H}_{2}$ be (separable) Hilbert spaces. We assume that these spaces are $\mathcal{Z}$ modules, where $\mathcal{Z}$ is an abelian von Neumann algbera (with a separable predual). Let us fix a faithful normal semifinte trace $\mu$ on $\mathcal{Z}$. We say that a vector $\xi \in \mathcal{H}_{1}$ is $\mu$-bounded if there exists a bounded operator $R^{\mu}(\xi)$ from $\mathcal{H}_{\mu}$ into $\mathcal{H}_{1}$ such that

$$
R^{\mu}(\xi) \eta_{\mu}(x)=x \xi
$$


for any $x \in \mathcal{N}_{\mu}$. Here $\mathcal{N}_{\mu}$ is an ideal of $\mathcal{Z}$ consisting of elements $x$ with $\mu\left(x^{*} x\right)<\infty$. Let $D\left(\mathcal{H}_{1}, \mu\right)$ denote the set of all $\mu$-bounded vectors in $\mathcal{H}_{1}$. Then the formula

$$
\left(\xi_{1} \otimes \eta_{1} \mid \xi_{2} \otimes \eta_{2}\right)=\left(R^{\mu}\left(\xi_{2}\right)^{*} R^{\mu}\left(\xi_{2}\right) \eta_{1} \mid \eta_{2}\right)
$$

defines a sesquilinear form on the algebraic tensor product $D\left(\mathcal{H}_{1}, \mu\right) \otimes \mathcal{H}_{2}$. We denote by $\mathcal{H}_{1} \otimes_{\mu} \mathcal{H}_{2}$ the Hilbert space obtained by completing $D\left(\mathcal{H}_{1}, \mu\right) \otimes \mathcal{H}_{2}$ modulo the set of vectors of length 0 , and call it the relative tensor product of $\mathcal{Z}$-modules $\mathcal{H}_{1}$ and $\mathcal{H}_{2}$ over $\mathcal{Z}$ with respect to $\mu$. Now let us consider the following situation. Suppose that we have two von Neumann algebras $\left\{\mathcal{M}_{1}, \mathcal{H}_{1}\right\}$ and $\left\{\mathcal{M}_{2}, \mathcal{H}_{2}\right\}$. We assume that $\mathcal{Z}$ is imbedded faithfully into both $\mathcal{M}_{1}$ and $\mathcal{M}_{2}$. Namely, those algeras are $\mathcal{Z}$-modules. Consequently, Hilbert spaces $\mathcal{H}_{1}$ and $\mathcal{H}_{2}$ are also $\mathcal{Z}$-modules. Thus we may form the relative tensor product $\mathcal{H}_{1} \otimes_{\mu} \mathcal{H}_{2}$ over $\mathcal{Z}$. Under this situation, there is a von Neumann algebra $\mathcal{M}_{1} * \mathcal{Z}_{2} \mathcal{M}_{2}$, called the fiber product of $\mathcal{M}_{1}$ and $\mathcal{M}_{2}$, on the Hilbert space $\mathcal{H}_{1} \otimes_{\mu} \mathcal{H}_{2}$ [S1]. Moreover, assume that both $\mathcal{M}_{1}$ and $\mathcal{M}_{2}$ admit normal representations, say $\pi_{1}$ and $\pi_{2}$, on Hilbert spaces $\mathcal{K}_{1}$ and $\mathcal{K}_{2}$ respectively. Through these representations, $\mathcal{K}_{i}$ 's become $\mathcal{Z}$-modules. So we get $\mathcal{K}_{1} \otimes_{\mu} \mathcal{K}_{2}$. Then we have a theorem due to Sauvageot [S2] which says that there exists a (unique) representation $\pi_{1} *_{\mathcal{Z}} \pi_{2}$ of $\mathcal{M}_{1} *_{\mathcal{Z}} \mathcal{M}_{2}$ on $\mathcal{K}_{1} \otimes_{\mu} \mathcal{K}_{2}$ satisfying some condition. These are the facts we need in order to show a Hopf-algebra-like structure of a groupoid von Neumann algebra.

Let $\left(\mathcal{G},\left\{\lambda^{x}\right\}, \Lambda, \delta\right)$ be a measured groupoid as before. From now on, $\mathcal{Z}$ denotes the abelian von Neumann algebra $L^{\infty}(X, \mu)$. This commutative algebra is faithfully imbedded into the groupoid von Neumann algbera $\mathcal{R}(\mathcal{G})$ via a mapping $h \in \mathcal{Z} \mapsto M(h \circ r) \in \mathcal{R}(\mathcal{G})$, where $M(f)$ stands for the multiplication by an essentially bounded measurable function 
f. As a consequence, both $\mathcal{R}(\mathcal{G})$ and $L^{2}(\mathcal{G}, \nu)$ become $\mathcal{Z}$-modules. So we may consider a fiber product von Neumann algebra $\mathcal{R}(\mathcal{G}) * \boldsymbol{z}(\mathcal{G})$ on a relative tensor product space $L^{2}(\mathcal{G}, \nu) \otimes_{\mu} L^{2}(\mathcal{G}, \nu)$. Then we have the following interesting theorem:

Theorem 2. There exists a normal *-isomorphism $\Gamma$ of $\mathcal{R}(\mathcal{G})$ into $\mathcal{R}(\mathcal{G}) *_{\mathcal{Z}} \mathcal{R}(\mathcal{G})$ which satisfies the identity:

$$
(\Gamma * \mathcal{Z} \iota) \circ \Gamma=(\iota * \mathcal{Z} \Gamma) \circ \Gamma
$$

where $\iota$ indicates the identity morphism of $\mathcal{R}(\mathcal{G})$.

It should be pointed out that the above identity is reminiscent of the coassociativity of a coproduct in the theory of Hopf algebra. We will make use of this morphism $\Gamma$ to define a coaction of a measured groupoid on a von Neumann algebra.

Definition 3 (Coactions). Let $\{\mathcal{N}, \mathcal{K}\}$ be a von Neumann algebra. Suppose that $\mathcal{N}$ is a $\mathcal{Z}$-module. Namely, $\mathcal{Z}=L^{\infty}(X, \mu)$ is faithfully imbedded into $\mathcal{N}$. Note that, in this case, $\mathcal{K}$ also becomes a $\mathcal{Z}$-module through the imbedding. Under this situation, a coaction of $\mathcal{G}$ on $\mathcal{N}$ is a $*$-isomorphism $\delta$ of $\mathcal{N}$ into the fiber product $\mathcal{N} *_{\mathcal{Z}} \mathcal{R}(\mathcal{G})$ on the Hilbert space $\mathcal{K} \otimes_{\mu} L^{2}(\mathcal{G}, \nu)$ satisfying

$$
\left(\delta *_{\mathcal{Z}} \iota\right) \circ \delta=(\iota * \mathcal{Z} \Gamma) \circ \delta
$$

where $\Gamma$ is the morphism appeared in the previous theorem.

It is obvious that these definitions 1 and 3 really generalize conventional definitions of group actions and group coactions. We should remark here that the notions of conjugacy 


\section{8}

and cocycle conjugacy of two actions (or conjugacy of two coactions) can be naturally introduced in this framework.

\section{$\S 2$. Crossed products by actions and coactions}

In the previous section, we introduce the concepts of an action and coaction of a measured groupoid which are considered as a natural generalization of what one finds in the group case. This section is concerned with a brief description of construction of a crossed product and the dual coaction or the dual action from a given action or coaction, respectively, of a measured groupoid.

Let $\left(\mathcal{G},\left\{\lambda^{x}\right\}, \Lambda, \delta\right)$ be a measured groupoid as before. Suppose that we are given a action $\left(\mathcal{G},\{\mathcal{M}(x)\},\left\{\alpha_{\gamma}\right\}\right)$ of $\mathcal{G}$. We keep all the notations introduced in the preceeding section. Now we briefly describe the construction of a crossed product algebra from the action. Let $\mathcal{H}(x)$ be the canonical $L^{2}$-space of the von Neumann algebra $\mathcal{M}(x)$ and $u(\gamma)$ be, as before, the canonical implementation of the ${ }^{*}$-isomorphism $\alpha_{\gamma}$. Thus $u(\gamma)$ is a unitary from $\mathcal{H}(s(\gamma))$ onto $\mathcal{H}(r(\gamma))$. By definition, $\{\mathcal{M}(x), \mathcal{H}(x)\}_{x \in X}$ is a measurable field of von Neumann algebras over $(X, \mu)$. Let $\{\mathcal{M}, \mathcal{H}\}$ be the von Neumann algebra obtained as the direct integral of the above field. Namely

$$
\mathcal{M}=\int_{X}^{\oplus} \mathcal{M}(x) d \mu(x), \quad \mathcal{H}=\int_{X}^{\oplus} \mathcal{H}(x) d \mu(x)
$$

For each $x \in X$, we set $\hat{\mathcal{H}}(x)=\mathcal{H}(x) \otimes L^{2}\left(\mathcal{G}^{x}, \lambda^{x}\right)$. Next we define a subspace $\hat{\mathcal{M}}(\gamma)$ of $\mathcal{L}(\hat{\mathcal{H}}(s(\gamma)), \mathcal{H}(r(\gamma)))$ by

$$
\hat{\mathcal{M}}(\gamma)=\{a u(\gamma) \otimes \lambda(\gamma): a \in \mathcal{M}(r(\gamma))\}
$$




\section{9}

where $\lambda(\gamma)$ is the left regular representation of $\mathcal{G}$ on the Hilbert bundle $\left\{L^{2}\left(\mathcal{G}^{x}, \lambda^{x}\right)\right\}_{x \in X}$ over $(X, \mu)$. Since $\{\hat{\mathcal{H}}(x)\}_{x \in X}$ is a measurable field of Hilbert spaces over $(X, \mu)$, we may form its direct integral:

$$
\int_{X}^{\oplus} \hat{\mathcal{H}}(x) d \mu(x)=\int_{X}^{\oplus} \mathcal{H}(x) \otimes L^{2}\left(\mathcal{G}^{x}, \lambda^{x}\right) d \mu(x)
$$

which is equal to the relative tensor product $\mathcal{H} \otimes_{\mu \mathcal{Z}} L^{2}(\mathcal{G}, \nu)$. Note that we can identify $\mathcal{H} \otimes_{\mu \mathcal{E}} L^{2}(\mathcal{G}, \nu)$ with the set of all functions $\eta$ from $\mathcal{G}$ into $\prod_{x \in X} \mathcal{H}(x)$ such that (i) $\eta(\gamma) \in$ $\mathcal{H}(r(\gamma)),(\gamma \in \mathcal{G})$. (ii) a function $x \in X \mapsto \int f_{m, x}(\gamma)\left(\xi_{n, x} \mid \eta(\gamma)\right) d \lambda^{x}(\gamma)$ is measurable for any $m, n \in \mathrm{N}$, where $\left\{\xi_{n}\right\}_{n \geq 1}$ and $\left\{f_{m}\right\}_{m \geq 1}$ are fundamental sequences of measurable fields $\{\mathcal{H}(x)\}$ and $\left\{L^{2}\left(\mathcal{G}^{x}, \lambda^{x}\right)\right\}$, respectively. (iii) $\int\|\eta(\gamma)\|^{2} d \nu(\gamma)<\infty$. The norm of such a function $\eta$ is defined by $\|\eta\|=\left(\int\|\eta(\gamma)\|^{2} d \nu(\gamma)\right)^{1 / 2}$.

We let $\mathcal{S}\left(\mathcal{G}, \prod_{\gamma \in \mathcal{G}} \hat{\mathcal{M}}(\gamma)\right)$ denote the set of all sections $A$ from $\mathcal{G}$ into $\prod_{\gamma \in \mathcal{G}} \hat{\mathcal{M}}(\gamma)$ with the following properties:

(1) If $\mathrm{A}$ is of the form $A(\gamma)=a(\gamma) u(\gamma) \otimes \lambda(\gamma)(a(\gamma) \in \mathcal{M}(r(\gamma)))$, then a function $\gamma \in \mathcal{G} \mapsto\left\langle a(\gamma), \omega_{r(\gamma)}>\right.$ is measurable for any $\omega=\int_{X}^{\oplus} \omega_{x} d \mu(x) \in \mathcal{M}_{*}=\int_{X}^{\oplus} \mathcal{M}(x)_{*} d \mu(x)$.

(2) The quantity $\|A\|_{H}=\max \left\{\|\lambda(\|A(\cdot)\|)\|_{\infty},\left\|\lambda\left(\left\|A^{\sharp}(\cdot)\right\|\right)\right\|_{\infty}\right\}$ is bounded, where $A^{\sharp}(\gamma)=\delta(\gamma)^{-1} A\left(\gamma^{-1}\right)^{*}$.

We will write $\mathcal{S}(\mathcal{M})=\mathcal{S}\left(\mathcal{G}, \prod_{\gamma \in \mathcal{G}} \hat{\mathcal{M}}(\gamma)\right)$ for short, if there is no danger of confusion. $\mathcal{S}(\mathcal{M})$ becomes a vector space under pointwise addtion and scalor multiplication. We can further equip $\mathcal{S}(\mathcal{M})$ with a $\sharp$-algerba structure. Its product $*$ and involution $\sharp$ are given by

$$
\begin{aligned}
(A * B)(\gamma) & =\int A\left(\gamma_{1}\right) B\left(\gamma_{1}^{-1} \gamma\right) d \lambda^{r(\gamma)}\left(\gamma_{1}\right) \quad(A, B \in \mathcal{S}(\mathcal{M})), \\
A^{\sharp}(\gamma) & =\delta(\gamma)^{-1} A\left(\gamma^{-1}\right)^{*} .
\end{aligned}
$$




\section{0}

Next we shall define a $\sharp$-*-preserving representation of the algebra $\mathcal{S}(\mathcal{M})$ on a Hilbert space $\mathcal{H} \otimes_{\mu} \mathcal{Z}^{2}(\mathcal{G}, \nu)$. It will be obtained by "integrating" each section in $\mathcal{S}(\mathcal{M})$.

Let $A$ be in $\mathcal{S}(\mathcal{M})$ and $\xi, \eta \in \mathcal{H} \otimes_{\mu \mathcal{Z}} L^{2}(\mathcal{G}, \nu)$. We may regard $\xi, \eta$ as functions on $\mathcal{G}$ as we observed before. The equation

$$
(\Phi(A) \xi \mid \eta)=\iint\left(a\left(\gamma_{1}\right) u\left(\gamma_{1}\right) \xi\left(\gamma_{1}{ }^{-1} \gamma\right) \mid \eta(\gamma)\right) d \lambda^{r(\gamma)}\left(\gamma_{1}\right) d \nu(\gamma)
$$

defines a bounded operator $\Phi(A)$ on $\mathcal{H} \otimes_{\mu \mathcal{Z}} L^{2}(\mathcal{G}, \nu)$, where $A$ has the form $A(\gamma)=$ $a(\gamma) u(\gamma) \otimes \lambda(\gamma), \quad a(\gamma) \in \mathcal{M}(r(\gamma))$ for any $\gamma \in \mathcal{G}$. It turns out that $\Phi$ is a nondegenerate norm decreasing $*$-representation of the algebra $\mathcal{S}(\mathcal{M})$ on $\hat{\mathcal{H}}=\mathcal{H} \otimes_{\mu} \mathcal{Z}^{2}(\mathcal{G}, \nu)$. The crossed product algebra of the action is by definition the weak closure of the nondegenerate *-algebra $\Phi(\mathcal{S}(\mathcal{M}))$ and is denoted by $\mathcal{M} \times{ }_{\alpha} \mathcal{G}$. It is shown in [Y] that there exists a coaction $\hat{\boldsymbol{\alpha}}$ of the groupoid on this new algebra which is called the dual coaction of the original action. Since the space is very limitted, we will not go into the details here. The author refers readers to $[\mathbf{Y}]$ for the detailed construction of the dual coaction.

Next we move on to the construction of a crossed product from a groupoid coaction. So we begin with a coaction $(\mathcal{G}, \mathcal{N}, \delta, \mathcal{K})$ of $\mathcal{G}$. Recall that $\delta$ is a ${ }^{*}$-isomorphism of $\mathcal{N}$ into the fiber product $\mathcal{N} *_{\mathcal{Z}} \mathcal{R}(\mathcal{G})$ on $\mathcal{K} \otimes_{\mu} L^{2}(\mathcal{G}, \nu)$ satisfying the identity: $\left(\delta *_{\mathcal{Z}} \iota\right) \circ \delta=$ $\left(\iota *_{\mathcal{Z}} \Gamma\right) \circ \delta$. We form a new von Neumann algebra on this Hilbert space $\mathcal{K} \otimes_{\mu} L^{2}(\mathcal{G}, \nu)$, generated by $\delta(\mathcal{N})$ and $\mathbf{C} \otimes{ }_{\mathcal{Z}} L^{\infty}(\mathcal{G}, \nu)$. We denote it by $\mathcal{N} \times{ }_{\delta} \mathcal{G}$ and call it the crossed product algebra of $\mathcal{N}$ by the coaction $\delta$. Note that, on $\mathcal{K} \otimes_{\mu} L^{2}(\mathcal{G}, \nu)$, there is an algebra $\left\{1 \otimes \mathcal{Z} M(h \circ s): h \in L^{\infty}(\mathcal{G}, \nu)\right\}$ which is a von Neumann subalgebra of $\mathbf{C} \otimes \mathcal{Z}^{\infty} L^{\infty}(\mathcal{G}, \nu)$. Hence $\left\{1 \otimes_{\mathcal{Z}} M(h \circ s): h \in L^{\infty}(\mathcal{G}, \nu)\right\}$ is contained in the crossed product $\mathcal{N} \times{ }_{\delta} \mathcal{G}$. Moreover, since $\mathcal{Z}_{S} \subseteq \mathcal{R}(\mathcal{G})^{\prime}$, we have that $1 \otimes_{\mathcal{Z}} M(h \circ s) \in \mathbf{C} \otimes_{\mathcal{Z}} \mathcal{R}(\mathcal{G})^{\prime} \subseteq \mathcal{N}^{\prime} \otimes_{\mathcal{Z}} \mathcal{R}(\mathcal{G})^{\prime}$ 
$=\left(\mathcal{N} *_{\mathcal{Z}} \mathcal{R}(\mathcal{G})\right)^{\prime}$. Form this together with the fact that $\delta(\mathcal{N}) \subseteq \mathcal{N} *_{\mathcal{Z}} \mathcal{R}(\mathcal{G})$, it follows that the algebra $\left\{1 \otimes_{\mathcal{Z}} M(h \circ s): h \in \mathcal{Z}\right\}$ is contained in the center $\mathcal{Z}\left(\mathcal{N} \times{ }_{\delta} \mathcal{G}\right)$ of the crossed product algebra $\mathcal{N} \times{ }_{\delta} \mathcal{G}$. Thus, $\mathcal{Z}=L^{\infty}(\mathcal{G}, \nu)$ can be regarded as a subalgebra of $\mathcal{Z}\left(\mathcal{N} \times{ }_{\delta} \mathcal{G}\right)$. We set $\mathcal{M}=\mathcal{N} \times{ }_{\delta} \mathcal{G}$ and $\mathcal{H}=\mathcal{K} \otimes_{\mu} L^{2}(\mathcal{G}, \nu)$. We then consider a decomposition of $\{\mathcal{M}, \mathcal{H}\}$ relative to $\mathcal{Z}$ :

$$
\mathcal{M}=\int_{X}^{\oplus} \mathcal{M}(x) d \mu(x), \quad \mathcal{H}=\int_{X}^{\oplus} \mathcal{H}(x) d \mu(x)
$$

We only assert without any proof that we can equip the measurable field $\{\mathcal{M}(x), \mathcal{H}(x)\}$ with a structure of an action of $\mathcal{G}$. The dual action $\hat{\delta}_{\gamma}$ is, roughly speaking, given by the adjoint of the "right regular representation of the measured groupoid $\mathcal{G}$ ".

\section{§ 3. Duality}

Now that, as we saw in the last section, we have a systematic way of associating a dual object with a given groupoid action or coaction, It is quite natural at this stage that, having these machineries in hand, one should ask himself whether a Takesaki duality type theorem holds in this groupoid setting. The answer is "Yes". We do have duality for actions and coactions of measured groupoids on von Neumann algebras. First we state the duality for groupoid actions.

Theorem 4 (Duality for actions). Suppose that $\left(\mathcal{G},\{\mathcal{M}(x)\}_{x \in X},\left\{\alpha_{\gamma}\right\}_{\gamma \in \mathcal{G}}\right)$ is an action of $\mathcal{G}$. Then the bidual action of the original one is conjugate to the action $\left(\mathcal{G},\left\{\mathcal{M}(x) \overline{\mathcal{L}}\left(L^{2}\left(\mathcal{G}_{x}, \lambda_{x}^{\prime}\right)\right)\right\}_{x \in X},\left\{\alpha_{\gamma} \otimes \operatorname{Ad} \rho(\gamma)\right\}_{\gamma \in \mathcal{G}}\right)$, where $\lambda_{x}^{\prime}$ is the Borel measure on $\mathcal{G}_{x}$ defined by the formula $\lambda_{x}^{\prime}(f)=\int f\left(\gamma^{-1}\right) \delta(\gamma) d \lambda^{x}(\gamma)$ for any Borel function $f$ on $\mathcal{G}_{x}$, and $\mathcal{L}(\mathcal{H})$ stands for the algbera of all bounded operators on a Hilbert space $\mathcal{H}$. 
Finally, $\rho(\gamma)$ is the unitary from $L^{2}\left(\mathcal{G}_{x}, \lambda_{x}^{\prime}\right)$ onto $L^{2}\left(\mathcal{G}_{y}, \lambda_{y}^{\prime}\right) \quad(\gamma: x \mapsto y)$ given by $\{\rho(\gamma) \xi\}\left(\gamma_{1}\right)=\delta(\gamma)^{1 / 2} \xi\left(\gamma_{1} \gamma\right)$

As a special case of this theorem, we obtain Nakagami-Takesaki duality for actions of second countable locally compact groups on von Neumann algebras.

Now we turn our attention to duality for a coaction of a measured groupoid. As we saw in the previous paragraph, duality holds good for a general measured groupoid in the case of an action. However, to prove duality for a coaction, we need to put a certain condition on it in order to have much control on the given coaction. The condition we will impose is called "integrability". Under this assumption, we can show the duality.

To define integrability of a coaction of a measured groupoid $\mathcal{G}$, we consider the following situation. Let $\{\mathcal{H}(x)=\mathcal{H}\}_{x \in X}$ be a constant field of Hilbert spaces over $X$. Suppose that we are given a family of $\{\mathcal{N}(\gamma)\}_{\gamma \in \mathcal{G}}$ of weakly closed subspaces of $\mathcal{L}(\mathcal{H})$ such that (1) $\mathcal{N}\left(\gamma_{1}\right) \mathcal{N}\left(\gamma_{2}\right) \subseteq \mathcal{N}\left(\gamma_{1} \gamma_{2}\right)$ whenever $\left(\gamma_{1}, \gamma_{2}\right) \in \mathcal{G}^{(2)},(2) \mathcal{N}(\gamma)^{*}=\mathcal{N}\left(\gamma^{-1}\right)$ for any $\gamma \in \mathcal{G}$. Note that, because of (1) and (2), each $\mathcal{N}(x)(x \in X)$ is a von Neumann algebra on $\mathcal{H}(x)=\mathcal{H}$. It should be remarked that this family of subspaces is reminiscent of a Banach *-algebraic bundle over a locally compact group in the sense of Fell (see [F] for the details). By "integrating" a suitable "section" of this bundle $\{\mathcal{N}(\gamma)\}_{\gamma \in \mathcal{G}}$ over $\mathcal{G}$, we obtain a bounded operator on an appropriate Hilbert space $\mathcal{K}$, which turns out to be a $\mathcal{Z}$-module. Let $\mathcal{N}$ be the von Neumann algebra consisting of operators obtained by integrating all possible such sections of the bundle. It can be shown that there exists a coaction $\delta$ of $\mathcal{G}$ on the algebra $\mathcal{N}$. Thus every bundle (or family) of subspaces over $\mathcal{G}$ with the above property gives rise to a coaction of $\mathcal{G}$ on a von Neumann algebra. We say 
that a coaction of a measured groupoid $\mathcal{G}$ on a von Neumann algebra is integrable if it arises from a bundle of subspaces of the above type. Now we are in a position to state duality for a coaction of a measured groupoid.

Theorem 5 (Duality for integrable coactions). Let $(\mathcal{G},\{\mathcal{N}, \mathcal{K}\}, \delta)$ be an integrable coaction of a measured groupoid $\mathcal{G}$. Then the bidual coaction of the original one is conjugate to a coaction $\left(\mathcal{G},\left\{\mathcal{N} *_{\mathcal{Z}} \mathcal{L}\left(L^{2}(\mathcal{G}, \nu)\right), \mathcal{K} \otimes_{\mu} L^{2}(\mathcal{G}, \nu)\right\}, \bar{\delta}\right)$, where $\bar{\delta}$ is a coaction determined by $\delta$.

Remark 6. In the definition of integrabilty of a coaction, we may replace the condition there by a weaker one that $\{\mathcal{H}(x)\}_{x \in X}$ is constant only on each equivalence class on $X$, that is, $\mathcal{H}(x)=\mathcal{H}(y)$ whenever $x \sim y$. Even if we relax the condition in this way, the above theorem still holds.

\section{$\S$ 4. Examples}

In this section, we give several interesting examples of actions and coaction.

Example 1. Let $\mathcal{G}$ be as before. For each $x \in X$, we set $\mathcal{M}(x)=\mathbf{C}(=$ the set of complex numbers). For each $\gamma \in \mathcal{G}$, we let $\alpha_{\gamma}: \mathbf{C}(s(\gamma)) \mapsto \mathbf{C}(r(\gamma))$ be the identity morphism. One can easily check that $\left(\mathcal{G},\{\mathcal{M}(x)\}_{x \in X},\left\{\alpha_{\gamma}\right\}_{\gamma \in \mathcal{G}}\right)$ is an action of $\mathcal{G}$. The crossed product algebra of the action turns out to be the groupoid von Neumann algebra $\mathcal{R}(\mathcal{G})$. The dual coaction is the coproduct $\Gamma$ of $\mathcal{R}(\mathcal{G})$ appeared in Theorem 2.

Example 2. By Theorem 2, the system $\left(\mathcal{G},\left\{\mathcal{R}(\mathcal{G}), L^{2}(\mathcal{G}, \nu)\right\}, \Gamma\right)$ forms a coaction of $\mathcal{G}$. From the duality theorem for actions of $\mathcal{G}$, together with the preceding example, it 


\section{4}

follows that $\left(\mathcal{G},\left\{\mathcal{L}\left(L^{2}\left(\mathcal{G}_{x}, \lambda_{x}^{\prime}\right)\right)\right\}_{x \in X},\{\operatorname{Ad} \rho(\gamma)\}_{\gamma \in \mathcal{G}}\right)$ is the dual system of the coaction in question.

Example 3. The following example is essentially due to A. Connes [C1] and T. Masuda $[M]$. We will just describe the situation briefly and not bother to go into the details. Let $\left(K, \mathbf{R}, F_{t}\right)$ be an ergodic (measure-preserving) smooth flow on a compact manifold $K$ with a probability measure. Then, by using an Anosov flow and a geodesic flow on a compact Riemann surface of constant negative sectional curvature, we can construct a locally compact (separable) measured groupoid $\mathcal{G}$ and its action $\left(\mathcal{G},\{\mathcal{M}(x)\}_{x \in X},\left\{\alpha_{\gamma}\right\}_{\gamma \in \mathcal{G}}\right)$ in such a way that the crossed product algbera of this action is an injective factor of type III, and that its smooth flow of weight is isomorphic to the given system $\left(K, \mathbf{R}, F_{t}\right)$. Thus, by using our machinery, we can systematically construct an injective factor of type III whose smooth flow of weight is a prescribed (ergodic) flow.

\section{$\S 5$. Concluding remark}

In writing this note, the author found that the assumption that $\mathcal{G}$ is a second countable locally compact topological groupoid is not essential for our argument. We only need $\mathcal{G}$ to be a groupoid with a standard Borel structure with respect to which all relevant maps and sets are Borel. With this new assumption, we are able to furnish a lot of interesting examples of groupoid actions. Those examples and a further investigation of groupoid actions and coactions will be published elsewhere in the near future.

\section{REFERENCES}


[C1] A. Connes, The von Neumann algebra of a foliation, Lecture Notes in Phys. Springer-Verlag 80 (1978).

[C2] - Sur la théorem non commutative l'intégration in Algébresd'opérateurs, Lecture Notes in Math., Springer-Verlag 725 (1979) 19-143.

[F] J.M.G. Fell, An extension of Mackey's method to Banach *-algebraic bundles, Memoirs Amer. Math. Soc. 90 (1969)

[J\&T] V.F.R. Jones and M. Takesaki, Actions of compact abelian groups on semifinite injective factors, Acta Math. 153 (1984) 213-258.

[K] H. Kosaki, Canonical $L^{p}$-spaces associated with an arbitrary abstract von Neumann algebra, Dissertation at UCLA (1980).

[M] T. Masuda, Groupoid dynamical systems and crossed product I-The case of $W^{*}$. systems, Publ. R.I.M.S. Kyoto Univ. 20 (1984) 929-957.

[N\&T] Y. Nakagami and M. Takesaki, Duality for crossed products of von Neumann algebras, Lecture Notes in Math. Springer-Verlag 731 (1979).

[Ra] A. Ramsay, Virtual groups and group actions, Adv. in Math. 6 (1971) 253-322.

[R] J. Renault, $A$ groupoid approach to $C^{*}$-algebras, Lecture Notes in Math. SpringerVerlag 793 (1980).

[S1] J.L. Sauvageot, Produits tensoriels de $\mathcal{Z}$-modules, Publ. Univ. P. \& M. Curie $n^{\circ} 23(1980)$.

[S2] — Produits tensoriels de $\mathcal{Z}$-modules et application, Lecture Notes in Math. Springer-Verlag 1132 (1983) 468-485.

[T] M. Takesaki, Duality for crossed products and the structure of von Neumann al- 


\section{6}

gebras of type III, Acta Math. 131 (1973) 249-310.

[Y] T. Yamanouchi, Duality for actions and coactions of measured groupoids on von Neumann algebras, Dissertation at UCLA (1990). 